\title{
Heart rate and ectopic prematurity in relation to sustained ventricular arrhythmias
}

\author{
SALIM YUSUF, ROBERTO LOPEZ, PETER SLEIGHT \\ From the Department of Cardiovascular Medicine, University of Oxford, Fohn Radcliffe Hospital, \\ Headington, Oxford
}

SUMMARY Forty-four patients with acute myocardial infarction were studied using 24-hour electrocardiographic monitoring. A linear correlation has been shown to exist between heart rate preceding accelerated idioventricular rhythm and ventricular tachycardia and the rates of these. The prematurity index was found to be inversely related to the preceding heart rate and also to the rate of accelerated idioventricular rhythm or ventricular tachycardia. Faster heart rates preceded extrasystoles with shorter prematurity indices and a significant inverse correlation was observed between preceding heart rate and prematurity index. Patients developing ventricular tachycardia had more frequent isolated ventricular extrasystoles and faster heart rates preceding the episode as compared with patients not showing ventricular tachycardia. No association was observed between ventricular tachycardia and accelerated idioventricular rhythm. All four patients developing ventricular fibrillation showed preceding ventricular tachycardia, with three of these patients demonstrating faster rates of ventricular tachycardia $(>170 / \mathrm{min})$ and shorter prematurity indices $(<0.80)$ than patients without ventricular fibrillation.

Heart rate may influence the severity of ventricular arrhythmia; the prematurity of an extrasystole is of importance in patients developing accelered idioventricular rhythm or ventricular tachycardia.

In an attempt to define rhythms predictive of ventricular fibrillation and ventricular tachycardia, several workers have identified "warning arrhythmias". The early ventricular extrasystole interrupting the $T$ wave has long been considered to be hazardous and a precursor of ventricular fibrillation..$^{1-3}$ In patients with acute myocardial infarction, antiarrhythmic therapy has been instituted when $R$ on $T$ extrasystoles have been identified. ${ }^{4}$ However, recent reports have challenged this view. ${ }^{56}$ Other reports suggest that faster heart rates, frequent isolated ventricular extrasystoles, and couplets predispose to ventricular tachycardia and ventricular fibrillation. ${ }^{2} 7$ However, the relation of the preceding heart rate to the prematurity of an extrasystole (prematurity index = coupling interval between preceding sinus complex and the first extrasystole/QT interval of the preceding sinus complex); and the relation of the prematurity index to the rate of the following ventricular arrhythmia has not been studied in patients with myocardial infarction.

* This work was supported by a grant from the British Heart Foundation.

Received for publication 18 January 1980
The purpose of this study was to investigate the following:

(i) The relation of the mean heart rate and the heart rate preceding a run of ventricular arrhythmia to the incidence and the rate of the ventricular arrhythmia and to the prematurity of the extrasystole starting the ventricular arrhythmia.

(ii) The relation between the prematurity index and the rate and incidence of ventricular tachycardia and accelerated idioventricular rhythm.

(iii) The association of a particular ventricular arrhythmia to other types of arrhythmia.

\section{Methods}

Forty-four patients admitted to the coronary care unit of the Radcliffe Infirmary with both electrocardiographic (recent ST segment elevation and $Q$ wave development) and enzyme evidence (at least twice normal values of creatine kinase) of acute myocardial infarction were studied at a mean time of six hours after the onset of chest pain. Thirty patients were studied within six hours, 11 between six and 12 hours, and three between 12 and 18 hours after the onset of chest pain. Twenty-four of these 
patients had anterior infarction and 20 had inferior infarction. The routine management was unaltered by the study procedure; diuretics, digoxin, and antiarrhythmic drugs were administered as indicated. A total of eight patients received lignocaine (100 $\mathrm{mg}$ as a bolus followed by an infusion of $4 \mathrm{mg} /$ min for the first two hours, $3 \mathrm{mg} / \mathrm{min}$ for the next two hours, and then $2 \mathrm{mg} / \mathrm{min}$ for a period of 20 hours) as antiarrhythmic therapy when ventricular fibrillation or frequent runs of ventricular tachycardia were observed by the nursing staff. Five patients at entry were already on beta-adrenergic blocking drugs for treatment of angina pectoris or hypertension. Nine patients received oral atenolol $100 \mathrm{mg}$ once daily as they were part of a prospective trial of beta-adrenergic blockade. No patient received beta-adrenergic blockers as antiarrhythmic therapy. Of the eight patients on lignocaine, five were also on beta-blockers so that $17 / 44$ were on lignocaine or beta-blockers.

All patients had normal serum levels of potassium and calcium throughout the entire study period.

DETECTION OF VENTRICULAR ARRHYTHMIAS In all patients we made continuous recordings of the electrocardiogram on the Oxford Instruments Medilog tape-recorder for the first $\mathbf{4 8}$ hours after admission. The records were then analysed on an Oxford Instruments Medilog electrocardiograph analysis system at 60 times real time. An observer always checked that the signals counted by the analysis system were accurate. In addition, the tape was then reanalysed by visual counting by the observer. Hourly counts of heart rate, isolated ventricular extrasystoles, couplets (two consecutive ventricular extrasystoles), triplets (three consecutive ventricular extrasystoles), episodes of accelerated idioventricular rhythm ( $>3$ consecutive ventricular extrasystoles at a rate less than 120 beats/min), and ventricular tachycardia ( $>3$ consecutive ventricular complexes at a rate $>120 / \mathrm{min}$ ) were recorded. Ventricular fibrillation occurred in only four of the 44 patients.

In most patients all isolated ventricular extrasystoles were recorded on paper. In a few patients with hourly rates of ventricular extrasystoles $>50$ /hour, sample recordings of ventricular extrasystoles during this period were obtained. In addition, all couplets, triplets, episodes of accelerated idioventricular rhythm, ventricular tachycardia, and ventricular fibrillation were recorded on paper. In cases of sustained accelerated idioventricular rhythm or ventricular tachycardia, the beginning and the end of the arrhythmia were recorded. These recordings were then analysed to ascertain:

(1) The prematurity index of the ventricular extrasystoles, couplets, triplets, accelerated idioventricular rhythm, ventricular tachycardia, and ventricular fibrillation.

(2) The heart rate preceding runs of accelerated idioventricular rhythm, ventricular tachycardia, and ventricular fibrillation from five consecutive sinus complexes.

(3) Rates of accelerated idioventricular rhythm and ventricular tachycardia.

\section{STATISTICAL METHODS}

Standard methods of correlation and regression, and paired and unpaired $t$ tests were used. All data are expressed as mean \pm 1 standard deviation.

\section{Results}

(A) HEART RATE

(i) Variation over 24 hours (Fig. 1)

A significant positive correlation was observed between the standard deviation and the mean of the individual hourly heart rates over the first 24 hours $(r=0.507 ; p<0.001)$ indicating greater variability in patients with higher heart rates.

(ii) Comparison between anterior and inferior infarcts Although the mean heart rate in patients with anterior infarction tended to be higher (73.89 $\pm 18.94)$ as compared with $67.58 \pm 13.36$ in patients with inferior infarction, this did not reach statistical significance $(p>0.05)$.

\section{(iii) Relation of mean heart rate to ventricular} arrhythmias

The mean heart rate averaged over 24 hours in

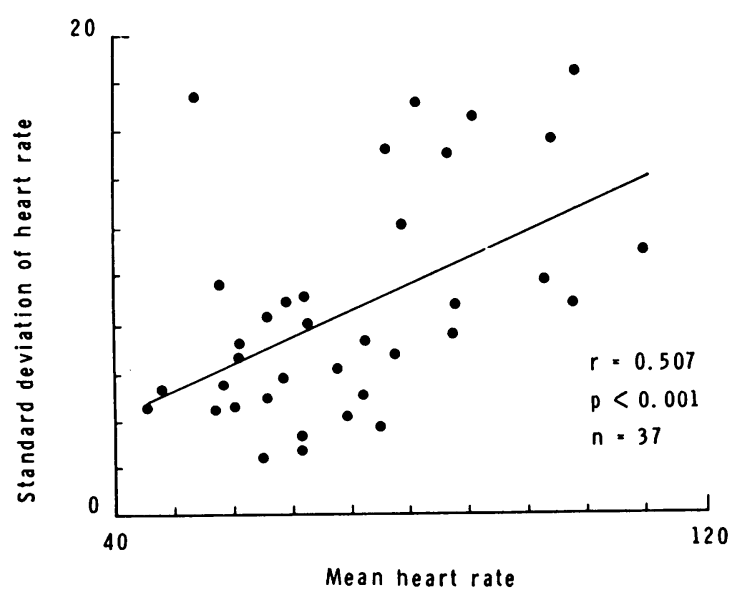

Fig. 1 Relation of the mean heart rate to the variation in heart rate. 
patients with ventricular tachycardia was not significantly different from those without ventricular tachycardia. Mean heart rate in patients with only accelerated idioventricular rate was $63 \pm 11.36$ as compared with $69 \pm 12.70$ in patients with accelerated idioventricular rate and ventricular tachycardia and $82.83 \pm 33.15$ in patients showing ventricular tachycardia alone. This trend, however, did not reach statistical significance.

(iv) Relation of preceding heart rate to rate of ventricular arrhythmias

A significant positive correlation between preceding heart rate and the rate of the accelerated idioventricular rate or ventricular tachycardia was observed when compared as continuous variables $(r=0.491 ; p<0.001)$. This relation was even more significant when accelerated idioventricular rate was considered separately $(r=0.696 ; \mathrm{p}<0.001 ; \mathrm{n}=52)$ (Fig. 2). In contrast, no significant relation between preceding sinus rate and the rate of ventricular tachycardia was observed $(r=0 \cdot 295, n=34)$ (Fig. 3). However, the heart rate preceding ventricular tachycardia was faster $(78.92 \pm 21.83)$ compared with the heart rate preceding accelerated idioventricular rate $(67.79 \pm 18.01)(2 p<0.02$; unpaired "t" test; Fig. 4).

\section{(v) Relation of preceding heart rate to prematurity} index (Fig. 5)

A significant inverse correlation between the heart rate preceding accelerated idioventricular rate and ventricular tachycardia and the prematurity index

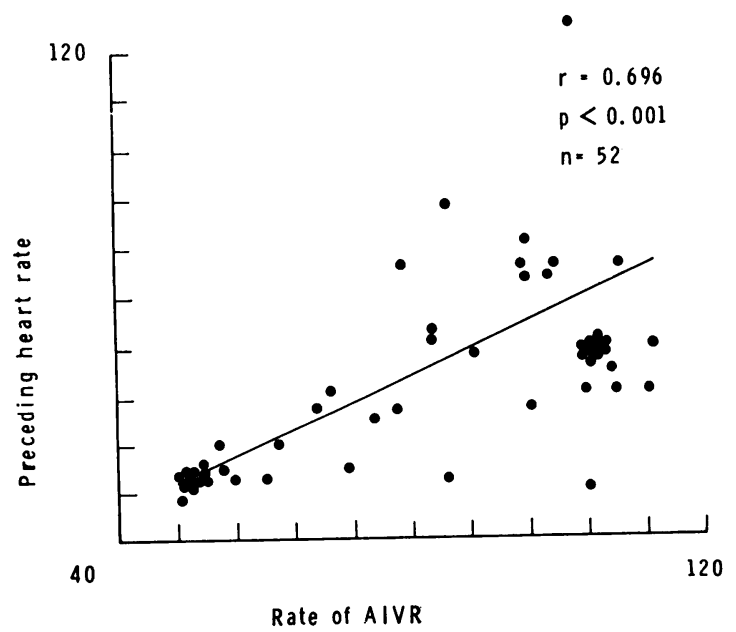

Fig. 2 Relation of the preceding heart rate to the rate of idioventricular rhythm (AIVR).

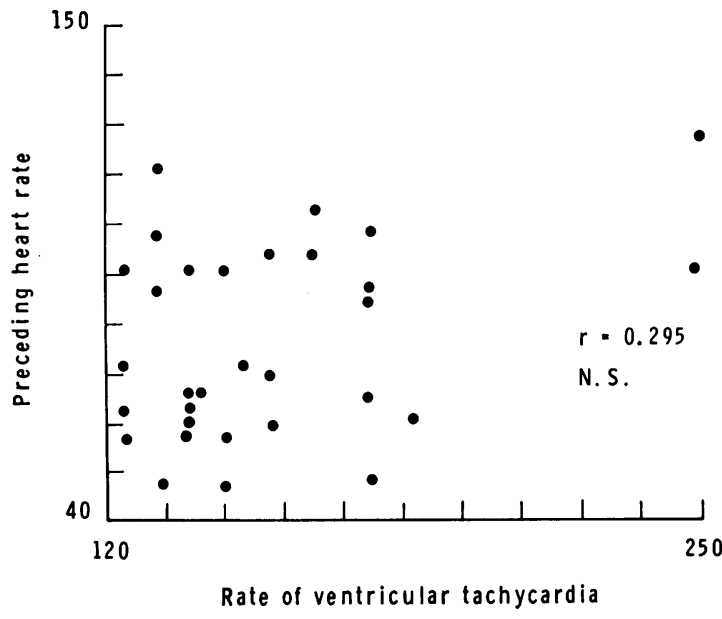

Fig. 3 Relation of the preceding heart rate to the rate of ventricular tachycardia.

of the first ventricular extrasystole was observed $(\mathrm{r}=-0.525 ; \mathrm{p}<0.001, \mathrm{n}=70)$.

(B) PREMATURITY INDEX

(i) Prematurity index and rate of ventricular arrhythmia

A significant inverse correlation between the prematurity index and the rate of the following accelerated idioventricular rate or ventricular tachycardia was observed $(r=-0.506 ; p<0.001)$ (Fig. 6). In addition, the prematurity index preceding accelerated idioventricular rate was significantly longer than the prematurity index preceding ventricular tachycardia $(1.89 \pm 0.53$ and $1.32 \pm 0.48$, respectively) $(2 \mathrm{p}<0.001)$ (Fig. 4$)$.

(ii) Prematurity index in patients with and without ventricular tachycardia

There was no significant difference when the prematurity index of both isolated ventricular extrasystoles and repetitive ventricular arrhythmia were compared in patients with and without ventricular tachycardia $(1.31 \pm 0.38$ in patients with ventricular tachycardia and $1.45 \pm 0.52$ in those without it).

(C) ISOLATED VENTRICULAR EXTRASYSTOLES Forty-three of the 44 patients studied had ventricular arrhythmias during the 24-hour period.

(i) Incidence of ventricular extrasystoles related to time after chest pain

The number of isolated ventricular extrasystoles decreased significantly from $75.68 \pm 122 \cdot 6$ in the 


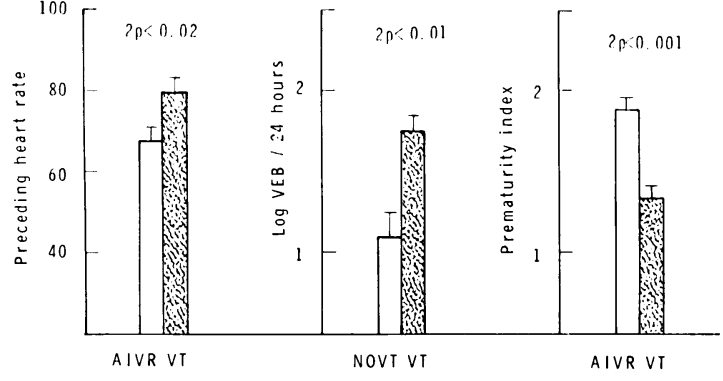

Fig. 4 Comparison of prematurity index, preceding heart rate, and the frequency of isolated ventricular extrasystole (VEB) in patients with accelerated idioventricular rhythm and ventricular tachycardia ( $A I V R$ and $V T)$.

first 24 hours to $40.98 \pm 87.25$ in the second 24 hours (paired " $t$ " test $2 p<0.05$ ).

(ii) Relation to incidence of accelerated idioventricular rate and ventricular tachycardia

Because the frequency of isolated ventricular extrasystole is not normally distributed, the data were expressed as logarithms before applying Student's " $\mathrm{t}$ " test. ${ }^{8}$ In patients showing ventricular tachycardia, the $\log _{10}$ ventricular extrasystole was 1.75 \pm 0.45 as compared with $1.11 \pm 0.87$ in patients without ventricular tachycardia $(2 p<0.01)$ (Fig. 4). In addition patients showing ventricular tachycardia and accelerated idioventricular rate had a significantly greater frequency of isolated ventricular extrasystoles as compared with patients showing neither rhythm $(1 \cdot 71 \pm 0.70$ in patients with

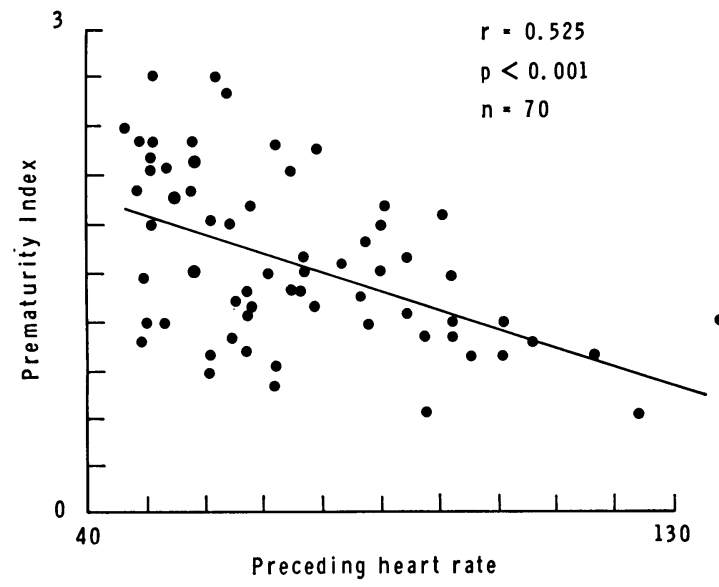

Fig. 5 Relation of preceding heart rate to the prematurity index. tachycardia and/or accelerated rhythm and $0.83 \pm$ 0.66 in patients without either) $(2 p<0.001)$. This suggests that patients with repetitive ventricular rhythms have more frequent isolated ventricular extrasystoles. This is even more pronounced in patients with ventricular tachycardia.

(D) COUPLETS AND TRIPLETS

The incidence of couplets and triplets was 55 per cent and 34 per cent, respectively. Though both tended to be higher in patients with ventricular tachycardia $(75 \%$ and $50 \%$, respectively) as compared with patients without ventricular tachycardia $(43 \%$ and $25 \%$ ), this was not statistically significant.

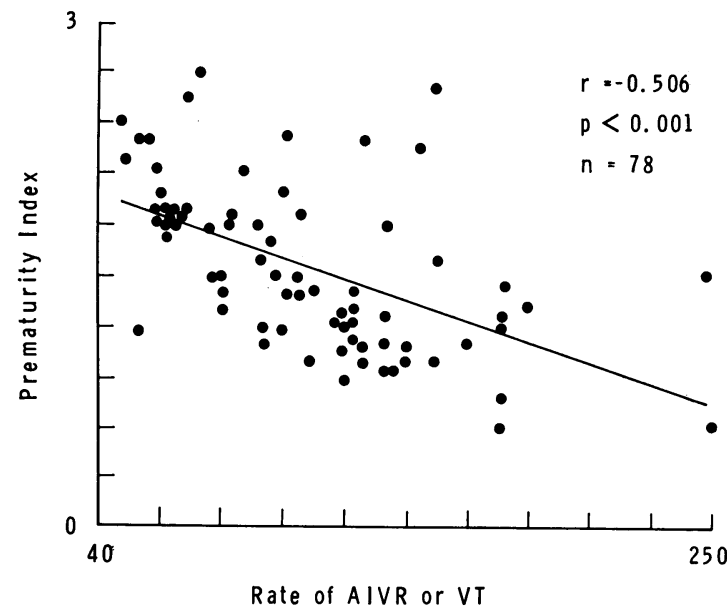

Fig. 6 Relation of prematurity index to the rate of ventricular arrhythmia ( $A I V R$ or VT).

(E) ASSOCIATION OF VENTRICULAR TACHYCARDIA AND ACCELERATED IDIOVENTRICULAR RHYTHM

We did not see any particular tendency for accelerated idioventricular rhythm and ventricular tachycardia to coexist in eight patients having both, eight with ventricular tachycardia only, and nine with accelerated idioventricular rhythm only.

\section{(F) ANALYSIS OF DATA WITH RELATION}

TO TIME

The relation of preceding heart rate to the prematurity index and to the rate of accelerated idioventricular rhythm or ventricular tachycardia was similar in the first and second 12 hours of recording, and in patients presenting within or after six hours of pain. Similarly, the relation between the prematurity index and the rate of 
accelerated idioventricular rhythm or ventricular tachycardia was independent of time.

(G) INFLUENCE OF DRUGS ON RELATION BETWEEN PRECEDING HEART RATE, PREMATURITY INDEX, AND RATE OF ACCELERATED IDIOVENTRICULAR RHYTHM AND VENTRICULAR TACHYCARDIA

Fourteen patients were on beta-blocking drugs during the period of tape-recording. The correlations between (1) preceding heart rate and prematurity index; (2) preceding heart rate and the rate of accelerated idioventricular rhythm and ventricular tachycardia; and (3) prematurity index and the rate of accelerated idioventricular rhythm and ventricular tachycardia were calculated in patients with and without beta-blocker treatment (Table). Similar correlations were found in all instances except one in the beta-blocker group, where no significant relation between preceding heart rate and the rate of accelerated idioventricular rhythm and ventricular tachycardia was observed. This suggests that the change in the prematurity index occurring at different heart rates is independent of any direct pharmacological action of betablockers and is probably related to heart rate changes per se.

Lignocaine was used in six patients within an hour of admission: four after ventricular fibrillation; and two for frequent ventricular tachycardia. In two other patients lignocaine was given after frequent runs of ventricular tachycardia were observed by the coronary care unit nurses approximately two hours after admission. This was continued for 24 hours as a continuous infusion and produced almost complete suppression of any ventricular tachycardia or accelerated idioventricular rhythm in these patients. It was therefore not possible to assess if the relation between preceding heart rate, prematurity index, and rate of sustained ventricular arrhythmia was altered by lignocaine.

Table Correlations between preceding heart rate, prematurity index, and rate of ventricular arrhythmia in patients treated and not treated with beta-blocker

\begin{tabular}{|c|c|c|}
\hline & $\begin{array}{l}\text { Patients on } \\
\text { beta-blocker }\end{array}$ & $\begin{array}{l}\text { Patients not on } \\
\text { beta-blocker }\end{array}$ \\
\hline $\begin{array}{l}\text { Preceding heart rate and } \\
\text { rate of ventricular arrhythmia }\end{array}$ & $\begin{array}{l}n=18 \\
r=0 \cdot 260 \\
N S\end{array}$ & $\begin{array}{l}\mathrm{n}=68 \\
\mathrm{r}=0.473 \\
\mathrm{p}<0.001\end{array}$ \\
\hline $\begin{array}{l}\text { Preceding heart rate } v \\
\text { prematurity index }\end{array}$ & $\begin{array}{l}r=-0.483 \\
p<0.05\end{array}$ & $\begin{array}{l}r=-0.673 \\
p<0.001\end{array}$ \\
\hline $\begin{array}{l}\text { Prematurity index } \mathrm{v} \text { rate } \\
\text { of ventricular rhythm }\end{array}$ & $\begin{array}{l}r=-0.687 \\
p<0.01\end{array}$ & $\begin{array}{l}r=-0.574 \\
p<0.001\end{array}$ \\
\hline
\end{tabular}

(H) CHARACTERISTICS OF PATIENTS WITH VENTRICULAR FIBRILLATION

Four patients developed ventricular fibrillation within an hour of admission and all four showed separate runs of ventricular tachycardia two to 30 minutes before the episode of ventricular fibrillation compared with only one of four patients showing accelerated idioventricular rhythm. Three patients had preceding ventricular tachycardia with rates 170,178 , and $178 / \mathrm{min}$. In one of these, an episode of ventricular tachycardia at $178 / \mathrm{min}$ deteriorated into ventricular fibrillation. The fourth patient had ventricular tachycardia at a rate of 138 / min. In comparison only two out of the $\mathbf{4 0}$ patients without ventricular fibrillation had ventricular tachycardia at rates exceeding $170 / \mathrm{min}$. In the first three patients the fibrillation was initiated by $\mathbf{R}$ on $\mathbf{T}$ ventricular extrasystoles (prematurity index $=0.62,0.62$, and 0.80 , respectively). The fourth episode of ventricular fibrillation was initiated by an extrasystole falling at the end of the descending limb of the $T$ wave (prematurity index $=0.90$ ). In comparison, no patient without fibrillation showed a prematurity index less than $0 \cdot 80$. Two patients without fibrillation showed $R$ on $T$ extrasystoles (prematurity index $=0.82$ and 0.84 ) one of whom also showed a run of ventricular tachycardia $(170 / \mathrm{min})$. Therefore patients with ventricular fibrillation showed faster ventricular tachycardia rates and earlier extrasystoles before the episode.

\section{Discussion}

Lown et al. ${ }^{4}$ defined a series of types of ventricular arrhythmia which were thought to be associated with an increased risk of ventricular fibrillation. Other workers have supported this original concept. ${ }^{9-11}$ Recent reports, however, do not support this and ventricular fibrillation has been reported to occur without warning arrhythmias..$^{12} 13$ In addition warning arrhythmias have been found to be equally common in patients with and without ventricular fibrillation. ${ }^{5} 714$ Since the incidence of ventricular fibrillation is relatively low in patients in hospital because of the delay in admission (20 out of 850 admissions in one series), ${ }^{15}$ many investigators ${ }^{5610}$ have looked at the precursors of ventricular tachycardia which are known to be associated with a high mortality. In our own study all four patients with ventricular fibrillation demonstrated ventricular tachycardia, with three having ventricular rates of $170 / \mathrm{min}$ or more before the episode. We have therefore studied "warning arrhythmias" before ventricular tachycardia occurs.

Like previous authors, ${ }^{5} 10$ we have observed a 
higher incidence of isolated ventricular extrasystoles in patients with ventricular tachycardia. Though couplets and triplets were more frequent in patients with ventricular tachycardia as compared with those without ventricular tachycardia, this difference did not reach statistical significance. We have also shown a significant decrease in the incidence of ventricular extrasystoles with increasing time from onset of chest pain, as the delay in the onset of taperecording would affect the probability of detecting warning arrhythmia. ${ }^{16}$ Further, Lie et al. ${ }^{7}$ have suggested that time after onset of chest pain should be an important criterion for the institution of antiarrhythmic therapy.

In this study we have chosen a new and quantitative approach to study the relation between preceding heart rate, prematurity index, and the rate of accelerated idioventricular rhythm and ventricular tachycardia. To use sharp distinctions of $R$ on $T$ and non-R on $T$ is not wholly accurate as there can be ventricular extrasystoles with widely differing prematurity indices in each category. Similarly the rate of ventricular tachycardia or accelerated idioventricular rhythm is more accurate than arbitrary definitions (about which there is no unanimity $)^{17}$ as the rates may vary within each category. We have, therefore, chosen to measure the individual prematurity index and relate it to the rate of the accompanying ventricular arrhythmia.

We have demonstrated a significant inverse correlation between the prematurity index and the rate of ventricular arrhythmia. However, Fig. 6 shows considerable scatter and it is evident that in some individual instances short prematurity index did not always lead to ventricular tachycardia. We have also shown that ventricular tachycardia is preceded by extrasystoles with a prematurity index significantly shorter than those preceding accelerated idioventricular rhythm. However, when we compared the prematurity index in patients with 'and without ventricular tachycardia, no significant difference was obtained, consistent with the observations of De Soyza et al..$^{5}$ and Chou and Wenzke. ${ }^{6}$ The possible reasons for this apparent discrepancy should be considered. The early studies of Smirk ${ }^{1}$ and Bleifer et al., though stressing that the $R$ on $T$ ventricular extrasystole was associated with a higher incidence of ventricular fibrillation and tachycardia, also showed that this did not always happen. It was Bleifer et al. ${ }^{2}$ who demonstrated that $R$ on $T$ extrasystoles alone did not carry a serious prognosis but that when seen in combination with repetitive ventricular arrhythmia they were dangerous. In fact, the majority of Smirk's patients showed both the $R$ on $T$ phenomenon and couplets, though this was not discussed in his paper. Further, Dolara ${ }^{18}$ has shown that the QT interval may be prolonged and when associated with a slightly premature extrasystole this gives the appearance of an $R$ on $T$; this may have a different significance. Recently Greene et al. ${ }^{19}$ have reported that artificial cardiac stimulation leading to repetitive ventricular activity in patients after myocardial infarction could identify patients prone to ventricular tachycardia and sudden death. It is possible that only in such patients whose ventricles are "vulnerable" to ventricular tachycardia is the $R$ on $T$ extrasystole of importance. In other patients it may not have the same significance.

Reports that late ventricular ectopic beats may initiate ventricular fibrillation and tachycardia do not necessarily contradict the role of the $R$ on $T$ extrasystole in their causation. Firstly, several factors (for example critical mass of myocardium available for re-entry, critical relation between conduction velocity and refractory period in certain parts of the myocardium, local myocardial electrolyte changes, disturbances of nervous regulation, etc.) other than an extrasystole falling in the "vulnerable period" may affect the response of the ventricle regarding the development of ventricular tachycardia and fibrillation..$^{20}$ Secondly, Waldo and Kaiser $^{21}$ have shown, using epicardial recordings, that localised fibrillation occurring in the infarct zone continues after the $T$ wave of the surface electrocardiogram and that this was often associated with the development of ventricular tachycardia and fibrillation. It may therefore be possible that ventricular extrasystoles occurring apparently after the $T$ wave on surface recordings may be occurring during the period of fragmented electrical activity when the ventricle may be "vulnerable".

We have shown a significant relation between preceding heart rate and the rate of sustained ventricular arrhythmia. This relation appears to be mainly for accelerated idioventricular rhythm and not for ventricular tachycardia. This observation supports the possibility that accelerated idioventricular rhythm is usually an escape rhythm. ${ }^{17}$ However, the heart rate preceding runs of ventricular tachycardia was significantly higher than that preceding accelerated idioventricular rhythm. Lie et $a .^{7}$ have reported a higher incidence of sinus tachycardia immediately preceding ventricular fibrillation. Recent experimental work ${ }^{22}$ has shown that a rapid heart rate may increase ventricular extrasystoles, non-homogeneity of refractory periods in contiguous areas of the myocardium, and vulnerability to ventricular fibrillation. In addition slow heart rates caused by vagal stimulation per se 
also increased ventricular fibrillation threshold. These considerations support our observations of a direct relation between heart rate and the rate of ventricular arrhythmia.

A significant inverse correlation between preceding heart rate and the prematurity of the ventricular ectopic has been shown. This may indicate that increasing heart rates may lead to ventricular ectopic beats with shorter prematurity index. The possibility that this may be an artefact must be considered. It is possible that as heart rate increases, and the RR interval decreases, the coupling interval of a manifest ventricular extrasystole may shorten correspondingly. However the QT interval also shortens with increasing heart rate and therefore any shortening of the prematurity index at fast heart rates is likely to be real and not artefact. However, the underlying mechanism for this relation is unclear and we are not aware of any work relating heart rates to the prematurity index.

We have shown that faster heart rates are associated with ventricular ectopic beats with shorter prematurity, which may in turn lead to faster ventricular arrhythmia in "susceptible" patients. This relation, however, may not exist in patients not susceptible to repetitive ventricular arrhythmias.

\section{References}

1 Smirk FH. R waves interrupting T waves. Br Heart $\mathcal{F}$ $1949 ; 11$ : 23-36.

2 Bleifer SB, Karpman HL, Sheppard JJ, Bleifer DJ. Relation between premature ventricular complexes and development of ventricular tachycardia. $\mathrm{Am} \mathfrak{F}$ Cardiol 1973; 31: 400-3.

3 Wiggers CJ, Wegria R, Pinera B. The effects of myocardial ischemia on fibrillation threshold: the mechanism of spontaneous ventricular fibrillation following coronary occlusion. Am $\mathcal{F}$ Physiol 1940; 131 : 309-16.

4 Lown B, Fakhro AM, Hood WB Jr, Thor GW. The coronary care unit. New perspectives and directions. $\mathcal{F} A M A$ 1967; 199: 188-98.

5 De Soyza N, Bissett JK, Kane JJ, Murphy ML, Doherty JE. Ectopic ventricular prematurity and its relationship to ventricular tachycardia in acute myocardial infarction in man. Circulation 1974; 50: 529-33.

6 Chou TC, Wenzke F. The importance of $R$ on $T$ phenomenon. Am Heart f 1978; 96: 191-4.

7 Lie KI, Wellens HJJ, Downar E, Durrer D. Observations on patients with primary ventricular fibrillation complicating acute myocardial infarction. Circulation 1975; 52: 755-9.

8 Ahumada GG, Karlsberg RP, Jaffe AS, Ambos HD, Sobel BE, Roberts R. Reduction of early ventricular arrhythmia by acebutolol in patients with acute myocardial infarction. Br Heart $\mathcal{F} 1979$; 41: 654-9.

9 Lawrie DM, Higgins MR, Godman MJ, Oliver MF, Julian DG, Donald KW. Ventricular fibrillation complicating acute myocardial infarction. Lancet 1968; ii: 523-8.

10 Mogensen L. Ventricular tachyarrhythmias and lignocaine prophylaxis in acute myocardial infarction. Acta Med Scand 1970; suppl: 513.

11 Julian DG, Valentine PA, Miller GG. Disturbances of rate, rhythm and conduction in acute myocardial infarction. A prospective study of 100 consecutive unselected patients with the aid of electrocardiographic monitoring. Am f Med 1964; 37: 915-27.

12 Bennett MA, Pentecost BL. Warning of cardiac arrest due to ventricular fibrillation and tachycardia. Lancet 1972; i: 1351-2.

13 Skjaeggestad O, Berstad A. Arrhythmias in the earliest phase of acute myocardial infarction. Acta Med Scand 1974; 196: 271-4.

14 El-Sherif N, Myerburg RJ, Scherlag BJ, et al. Electrocardiographic antecedents of primary ventricular fibrillation. Value of the R-on- $T$ phenomenon in myocardial infarction. $B r$ Heart $\mathcal{F} 1976$; 38: 415-22.

15 Dhurandhar RW, MacMillan RL, Brown KWG. Primary ventricular fibrillation complicating acute myocardial infarction. $A m \mathcal{F}$ Cardiol 1971; 27: 347-51.

16 Campbell RWF, Murray A. Significance of arrhythmias in the early and late phase of myocardial infarction. In Hjalmarson $\AA$, Wilhelmsen $\mathrm{L}$, eds. Acute and long-term medical management of myocardial ischaemia. Mölndal, Sweden: Hässle, 1978: 130-6.

17 Bigger JT Jr, Dresdale RJ, Heissenbuttel RH, Weld FM, Wit AL. Ventricular arrhythmias in ischemic heart disease: mechanism, prevalence, significance, and management. Prog Cardiovasc Dis 1977; 19 : 255-300.

18 Dolara A. Early premature ventricular beats, repetitive ventricular response, and ventricular fibrillation. Am Heart $\mathcal{f}$ 1967; 74: 332-6.

19 Greene HL, Reid PR, Schaeffer AH. The repetitive ventricular response in man: a predictor of sudden death. $N$ Engl f Med 1978; 299: 730-4.

20 Surawicz B. Ventricular fibrillation. Am $\mathcal{f}$ Cardiol 1971 ; 28: 268-87.

21 Waldo AL, Kaiser GA. A study of ventricular arrhythmias associated with acute myocardial infarction in the canine heart. Circulation 1973; 47: 1222-8.

22 Kent KM, Smith ER, Redwood DR, Epstein SE. Electrical stability of acutely ischemic myocardium: influences of heart rate and vagal stimulation. Circulation 1973; 47: 291.

Requests for reprints to Dr S Yusuf, Department of Cardiovascular Medicine, John Radcliffe Hospital, Headington, Oxford OX3 9DU. 